\title{
Evolution of haplont, diplont or haploid-diploid life cycles when haploid and diploid fitnesses are not equal
}

\author{
Michael F Scott ${ }^{1}$, Marie Rescan ${ }^{2,3}$ \\ ${ }^{1}$ Department of Botany, University of British Columbia, 3529-6270 Univer- \\ sity Boulevard, Vancouver, BC, Canada V6T 1 Z4 \\ ${ }^{2}$ CNRS, Unité Mixte Internationale 3614, Evolutionary Biology and Ecology \\ of Algae, Roscoff, France \\ ${ }^{3}$ Sorbonne Universités, Université Pierre et Marie Curie, University of Paris \\ 6, Roscoff, France \\ email: mfscott@biodiversity.ubc.ca. \\ Keywords: alternation of generations, life cycle evolution, diplohaplontic, \\ modifier model, multilocus simulations \\ Running Title: Haploid-Diploid Evolution
}




\begin{abstract}
Many organisms spend a significant portion of their life cycle as haploids and as diploids (a haploid-diploid life cycle). However, the evolutionary processes that could maintain this sort of life cycle are unclear. Most previous models of ploidy evolution have assumed that the fitness effects of new mutations are equal in haploids and homozygous diploids, however, this equivalency is not supported by empirical

data. With different mutational effects, the overall (intrinsic) fitness of a haploid would not be equal to that of a diploid after a series of substitution events. Intrinsic fitness differences between haploids and diploids can also arise directly, e.g., because diploids tend to have larger cell sizes than haploids. Here, we include intrinsic fitness differences into genetic models for the evolution of time spent in the haploid versus diploid phases, in which ploidy affects whether new mutations are masked. Life cycle evolution can affected by intrinsic fitness differences between phases, the masking of mutations, or a combination of both. We find parameter ranges where these two selective forces act and show that the balance between them can favour convergence on a haploid-diploid life cycle, which is not observed in the absence of intrinsic fitness differences.
\end{abstract}




\section{Introduction}

22 Sexual reproduction in eukaryotes requires an alternation of haploid and diploid phases in the life cycle. Across taxa, there is a great deal of variation

24

28

30

36

in the amount of growth (and time spent) in each of the haploid and diploid phases (see Valero et al. 1992, Klinger 1993, Richerd et al. 1993, Bell 1994; 1997, Mable and Otto 1998, Coelho et al. 2007). Some organisms, including almost all animals, are diplontic (somatic development occurs only in the diploid phase) and others, including dictyostelid slime moulds, and some green algae (e.g., Chara), are haplontic (somatic development occurs only in the haploid phase). However, a large and phylogenetically diverse group of eukaryotes, including most land plants, basidiomycete fungi, most brown algae, red algae and some green algae, undergo some mitotic growth in both the haploid and diploid phases, which is referred to as a haploid-diploid 34 life cycle here (sometimes called diplohaplontic or haplodiplontic) to avoid confusion with arrhenotoky ('haplodiploid' sex determination). While several theoretical studies have explored the conditions that should favour expansion of the haploid or diploid phases, there are still relatively few studies that show how a haploid-diploid life cycle could be maintained by selection.

A prominent theory for the evolution of either haplont or diplont life cycles involves the direct consequences of ploidy level on the expression of deleterious mutations. The fitness effects of a deleterious mutation can be partially hidden by the homologous gene copy in diploids, which is favourable if a heterozygote has a higher fitness than the average fitness of the two com44 ponent haploids. Thus modifier models, in which the extent of haploid and diploid phases is determined by a second locus, have found that diplonty is favoured when deleterious mutations are partially recessive and haplonty is favoured when deleterious mutations are partially dominant (Perrot et al. 1991, Otto and Goldstein 1992, Jenkins and Kirkpatrick 1994; 1995). As a consequence of mutations being partially concealed, an expanded diploid phase allows mutations to reach a higher frequency and thus increases muta- 
tion load (Crow and Kimura 1965, Kondrashov and Crow 1991). Modifiers

that expand the diploid phase therefore become associated with lower quality genetic backgrounds. These associations are broken apart by recombination and so diplonty is favoured over a wider parameter range when recombination rates are higher (Otto and Goldstein 1992).

56 The evolution of life cycles in sexual organisms appears to be similarly influenced by beneficial mutations. Using a numerical simulation approach, Otto (1994) and Orr and Otto (1994) show that diplonty is favoured during sweeps of beneficial mutations that are partially dominant. Increasing the length of the diploid phase of the life cycle increases the amount of selection experienced by heterozygotes and, with partial dominance, heterozygotes have higher fitness than the average fitness of the two component haploids. Conversely, haplonty is favoured when beneficial mutations are partially recessive. Again, lower recombination rates between the life cycle modifier and beneficial mutations broaden the parameter range over which haplonty is favoured because of associations between the modifiers expanding the haploid phase and higher quality genetic backgrounds that evolve when beneficial mutations are not masked.

These models typically assume that the overall fitness of haploids or diploids is the same. However, even with identical genomes, haploid and diploid cells typically differ in size and often in shape (e.g., Mable 2001), and growth and survival often differs between haploid and diploid phases. The phase with higher fitness and the magnitude of fitness differences varies widely and is heavily dependent on environmental context (Mable and Otto 1998, Thornber 2006). In Saccharomyces yeast, differences between haploid and diploid growth rates measured by Zörgö et al. (2013) range from being negligible to substantial (one phase can have growth rates up to 1.75 times higher) in different environments. Similar differences in growth rate and survival are observed between haploid and diploid phases of the red algae Gracilaria verrucosa and Chondracanthus squarrulosus in some laboratory 
conditions (Destombe et al. 1993, Pacheco-Ruíz et al. 2011). In addition,

the fitness effect of new mutations may be unequal when present in haploids or in homozygous diploids, as reported by Gerstein (2012) and Zörgö et al. (2013). Therefore, following a series of substitution events, the overall (intrinsic) fitness of a haploid and a diploid should not be equal, as explored here.

The models discussed above assume that selection is independent of the densities of haploid and diploid individuals. These models also predict that either haplonty or diplonty evolves but not biphasic, haploid-diploid life cycles. Hughes and Otto (1999) and Rescan et al. (2016) consider densitydependent selection in which haploids and diploids occupy different ecological niches and show that haploid-diploid life cycles can evolve in order to exploit both the haploid and diploid ecological niches. In this study, we complement these studies by considering only density independent selection in order to focus on intrinsic fitness differences between haploids and diploids.

The effect of intrinsic fitness differences on the evolution of the life cycle may seem obvious - selection should favour expansion of whichever phase (haploid or diploid) has higher fitness, as found by Jenkins and Kirkpatrick (1994; 1995). However, Jenkins and Kirkpatrick (1994; 1995) only considered the case where the differences in intrinsic fitness is either much larger or much smaller than the genome-wide deleterious mutation rate. Here, we consider the case where the two forces are of similar strength and quantify the parameters (e.g., mutation rate) for which this is true. In addition, we consider the effect of beneficial mutations on life cycle evolution when there are intrinsic fitness differences between haploids and diploids. We show that haploid-diploid life cycle can evolve even in the absence of density dependent selection due to a balance between intrinsic fitness differences between phases and the genetic effects of masking/revealing mutations. We also consider branching conditions and find that, in haploid-diploid populations, sexually interbreeding mixtures of haploid and diploid specialists can be favoured (see 
also Rescan et al. 2016).

${ }_{112}$ Model

We consider life cycle evolution using a modifier model in which the propor-

114 tion of time spent in the haploid and diploid phases depends on the genotype at a modifier locus. Selection on the modifier results from viability selection on a set of $L$ other loci. We first present a two-locus model, in which there is one viability locus and one modifier locus. We then extrapolate our results to the evolution of a modifier locus linked to many loci under selection; selection on a modifier caused by many loci is well approximated by the sum of the selective effect of each pairwise interaction considered separately (e.g., Jenkins and Kirkpatrick 1995, Otto and Bourguet 1999, Hough et al. 2013), assuming that the viability loci are loosely linked, autosomal and nonepistatic and the modifier has a small effect. We then test this approach by comparing our results to an explicit multi-locus simulation. Finally, we show that beneficial mutations can generate selection on the life cycle similar to that caused by deleterious mutations.

\section{Analytical Model}

In the modifier model presented here (figure 1b), zygotes are formed during synchronous random mating. The diploid genotype $(i j)$ at the modifier locus ( $M M, M m$, or $\mathrm{mm}$ ) determines the timing of meiosis and hence the proportion of time each individual spends as a diploid $\left(1-t_{i j}\right)$ and as a haploid $\left(t_{i j}\right)$. Here, $S_{h}$ and $S_{d}$ represent selection acting across the genome due to intrinsic fitness differences between haploids and diploids. As our initial focus will be on the selection experienced at each of $L$ selected loci, we also define $\sigma_{h}=S_{h} / L$ and $\sigma_{d}=S_{d} / L$ as the intrinsic fitnesses per viability locus. When $\sigma_{h}>\sigma_{d}$, haploids have higher fitness than diploids and the fitness of diploids is higher when $\sigma_{d}>\sigma_{h}$. At each viability locus, we consider a wild type and 
mutant allele (alleles $A$ and $a$ ). The mutant allele at each viability locus, $a$, can have a different effect on fitness when present in a haploid $\left(s_{h}\right)$ or in a homozygous diploid $\left(s_{d}\right)$. The fitness of heterozygous diploids depends on the dominance of these mutations, given by $h$. When considering deleterious mutations, $s_{h}$ and $s_{d}$ are both negative, and when considering beneficial mutations, $s_{h}$ and $s_{d}$ are both positive. The fitnesses of the various genotypes are given in table 1 . Recombination between the modifier and viability locus (at rate $r$ ) and mutation (from $A$ to $a$, at rate $\mu$ per viability locus) occur at meiosis followed by haploid selection and then gamete production. The frequencies of genotypes $M A, M a, m A$ and $m a$ are censused in the gametes (given by $x_{1}, x_{2}, x_{3}$ and $x_{4}$ respectively).

Table 1: Fitnesses of different genotypes.

\begin{tabular}{ll}
\hline \hline Genotype & Fitness \\
\hline$A$ & $w_{A}\left(t_{i j}\right)=\exp \left[t_{i j} \sigma_{h}\right]$ \\
$a$ & $w_{a}\left(t_{i j}\right)=\exp \left[t_{i j}\left(\sigma_{h}+s_{h}\right)\right]$ \\
$A A$ & $w_{A A}\left(t_{i j}\right)=\exp \left[\left(1-t_{i j}\right)\left(\sigma_{d}\right)\right]$ \\
$A a$ & $w_{A a}\left(t_{i j}\right)=\exp \left[\left(1-t_{i j}\right)\left(\sigma_{d}+h s_{d}\right)\right]$ \\
$a a$ & $w_{a a}\left(t_{i j}\right)=\exp \left[\left(1-t_{i j}\right)\left(\sigma_{d}+s_{d}\right)\right]$ \\
\hline
\end{tabular}

Previous models have made various different life cycle assumptions, summarized in table 2. In 'discrete selection' models, selection occurs once per generation and modifiers affect whether selection occurs during the haploid or diploid phase, figure 1a. On the other hand, 'continuous selection' models assume that selection occurs continuously throughout the life cycle, figure $1 \mathrm{~b}$. In addition, some models have assumed that mutations occur upon gamete production, and others assume that mutations occur at meiosis. Thus, there are four possible life cycles, recursion equations for these different life cycles are provided in the appendix. Generally, our results are unaffected by using these alternative models, these analyses can be found in the supplementary Mathematica file (Wolfram Research Inc. 2010). However, there are two cases in which life cycle assumptions qualitatively impact results. 
Table 2: Life cycle assumptions used in various modifier models.

\begin{tabular}{l||cc}
\multicolumn{1}{l||}{} & $\begin{array}{c}\text { Mutations at } \\
\text { Gamete Production }\end{array}$ & \multicolumn{1}{c}{$\begin{array}{c}\text { Mutations at } \\
\text { Meiosis }\end{array}$} \\
\hline \hline \multirow{2}{*}{$\begin{array}{l}\text { Discrete Selection } \\
\text { (Figure 1a) }\end{array}$} & $\begin{array}{l}\text { Perrot et al. (1991) } \\
\text { Otto and Goldstein (1992) } \\
\text { Otto and Marks (1996) }\end{array}$ & Hall (2000) \\
& Rescan et al. (2016) & Orr and Otto (1994) \\
Continuous Selection & Otto $(1994)^{a}$ & Otto (1994) \\
(Figure 1b) & & Jenkins and \\
& & Kirkpatrick $(1994 ; 1995)$
\end{tabular}

162

${ }^{a}$ Otto (1994) allows mutations to occur at both gamete production and meiosis

Firstly, Hall (2000) showed that 'polymorphic' haploid-diploid life cycles can evolve if mutations occur at meiosis and selection is discrete. This life cycle allows diploids to escape selection on new mutations for one generation, generating an advantage to diploids, which allows convergence to occur when deleterious mutations favour haploids. As shown below, meiotic mutation does not favour haploid-diploid life cycles in the continuous selection model (figure 1b) because diploids do not escape selection on new mutations.

Secondly, alternative mating schemes have previously only been considered by Otto and Marks (1996), who assume discrete selection and mutations at gamete production (and no differences in intrinsic fitness between haploids and diploids). They found that haploidy is favoured over a larger parameter range when selfing, asexual reproduction or assortative mating is common. In the appendix, we include selfing into all four life cycle models and show that this conclusion only applies when the fitness of haploids and homozygous diploids are assumed to be equal (e.g., no intrinsic fitness differences) because selfing increases homozygosity. Furthermore, the conclusions of Otto and Marks (1996) require that mutations occur at gamete production, see 
appendix.

180

\section{Multilocus Simulations}

We used individual-based simulations $(\mathrm{C}++$ program available in the Dryad Digital Repository) to test predictions from our analytical model when deleterious mutations segregate at $L$ loci. Each individual carries either one or two copies of a chromosome (depending on its ploidy level) represented by a modifier locus (located at the midpoint of the chromosome) and a sequence of $L$ bits (0 or 1$)$ corresponding to the different loci.

Mutations occur at a rate $U$ per generation: the number of new mutations per chromosome is sampled from a Poisson distribution with parameter $U$ and distributed randomly across the genome; alleles at mutant loci are switched from 0 to 1 or from 1 to 0 . Mutation and back mutation thus occur at the same rate, but back mutations should generally have negligible effects under the parameter values that we use, as deleterious alleles remain at low frequencies. We assume that all deleterious alleles have the same effects on fitness $\left(s_{d}, s_{h}\right.$, and $h$ are constant) and that these effects multiply across loci: the fitness of a haploid carrying $\mathrm{n}$ deleterious alleles is given by $w_{h}=$ $\exp \left[S_{h}+s_{h} n\right]$, while the fitness of a diploid carrying $n_{h e}$ deleterious alleles in the heterozygous state, and $n_{h o}$ in the homozygous state is given by $w_{d}=$ $\exp \left[S_{d}+n_{h e} h s_{d}+n_{h o} s_{d}\right]$.

At the start of each generation, all $\mathrm{N}$ individuals are diploid. To produce the $2 \mathrm{~N}$ gametes that will form the diploids of the next generation, a diploid individual is sampled randomly among all diploids of the previous generation, and undergoes meiosis to produce a haploid; the number of cross-overs is sampled from a Poisson distribution with parameter $R$, while the position of each cross-over is sampled from a uniform distribution. If a random number sampled from a uniform distribution between 0 and 1 is lower than $w_{d}^{1-t} w_{h}^{t}$ (where $w_{d}$ and $w_{h}$ are the fitnesses of the diploid parent and haploid offspring), divided by its maximal possible value, then the haploid is 


\section{Deleterious Mutations}

232 fulfilled. circles).

\section{Results}

retained; otherwise another diploid parent is sampled, until the condition is

At the beginning of the simulation, the modifier locus is fixed for an allele coding for an initial length of the haploid phase $t_{\text {init }}$ (all simulations were performed for $t_{\text {init }}$ values of $0.1,0.5$ and 0.9 ) and all selected loci are fixed for allele 0 . Then, deleterious mutations are introduced at rate $\mathrm{U}$ per chromosome (the length of the haploid phase being still fixed to $t_{\text {init }}$ ) until the population reaches mutation-selection equilibrium (after generally 2,000 generations). After that, mutations at the modifier locus are introduced at a rate $m_{M}$ per generation. When a mutation occurs, the length of the haploid phase coded by the mutant allele is sampled from a uniform distribution between $t_{\text {old }}-0.1$ and $t_{\text {old }}+0.1$, where $t_{\text {old }}$ is the value of the parent allele; if the new value is negative or higher than 1 , it is set to 0 or 1 , respectively. We assume additivity among modifier alleles such that a zygote with alleles $t_{1}$ and $t_{2}$ will have a haploid phase of length $t=\left(t_{1}+t_{2}\right) / 2$. Simulations initially lasted 100,000 generations, which was sufficient in most cases for the average rate of diploidy to reach steady state, $\bar{t}$. We categorized the life cycle that evolved at the end of the simulation as haplont $(\bar{t}>0.9$, white circles in figures 2 and $3 \mathrm{~b})$, diplont $(\bar{t}<0.1$, black circles), or haploid-diploid $(0.1<\bar{t}<0.9$, green circles $)$. In some cases, there was a repelling state such that the population evolved to haplonty or diplonty depending on $t_{\text {init }}$ (red

We first find the frequency of deleterious mutations at mutation-selection balance $\left(\hat{q}_{a}\right)$ when the modifier locus is fixed for a particular resident allele ( $M M$ fixed, so that the length of the haploid phase is $t_{M M}$ ). Assuming that the per locus mutation rate $(\mu)$ is small, terms of the order of the square of 
the per locus mutation rate can be ignored, yielding

$$
\hat{q}_{a}=\frac{\mu \exp \left[t_{M M} s_{h}\right]}{1-\exp \left[t_{M M} s_{h}+\left(1-t_{M M}\right) h s_{d}\right]},
$$

assuming there is some haploid or diploidy heterozygous expression so the denominator isn't near zero. When deleterious mutations are partially masked by the homologous gene copy in diploids $\left(h s_{d} / s_{h}<1\right)$, the frequency of deleterious mutations $\left(\hat{q}_{a}\right)$ is higher when the diploid phase is longer (lower $\left.t_{M M}\right)$.

Life cycle evolution is considered by introducing an allele $(m)$ at the modifier locus that controls the timing of meiosis and evaluating whether its frequency increases when rare. Mutants are able to invade when the leading eigenvalue of the system described by equations A.1c and A.1d, $\lambda_{l}$, is greater than one. Jenkins and Kirkpatrick (1994) derive a version of $\lambda_{l}$ when $s_{d}=s_{h}$, however, they only discuss per locus intrinsic fitness differences that are of a much greater magnitude than the mutation load $\left(\left|\sigma_{d}-\sigma_{h}\right| \gg \mu\right)$. To investigate the interaction between these selective forces we first present an approximation of $\lambda_{l}$ in which the per locus fitness difference between haploids and diploids $\left(\left|\sigma_{d}-\sigma_{h}\right|\right)$ is of similar magnitude to the per locus mutation rate, $O\left(\epsilon^{2}\right)$, the selective disadvantage of mutants $\left(s_{d}\right.$ and $\left.s_{h}\right)$ is of a larger order of magnitude, $O(\epsilon)$, and linkage is loose $(r$ of $O(1))$ yielding

$$
\lambda_{l} \approx 1+\left(t_{M m}-t_{M M}\right)\left(\sigma_{h}-\sigma_{d}+2\left(-s_{h}\right) \hat{q}_{a}\left(\frac{h s_{d}}{s_{h}}-\frac{1}{2}\right)\right)+O\left(\epsilon^{3}\right) .
$$

254 Because mutation rates are small, deleterious mutations are found at low frequencies, therefore life cycle evolution depends only on the fitness of heterozygous mutants and not homozygous mutants (i.e., $s_{d}$ is always found with the dominance coefficient, $h$ ). Consequently, life cycle evolution depends only on the 'effective dominance', $h_{e}=h s_{d} / s_{h}$, rather than dominance per se. 
Life cycle modifiers affect the amount of selection heterozygous zygotes will subsequently experience as heterozygous diploids versus as the component haploid genotypes. Heterozygous diploids have higher fitness than the average of the two component haploids when deleterious mutations are effectively partially recessive $\left(0<h s_{d} / s_{h}<1 / 2\right)$, favouring diploidy. Conversely, effectively partially dominant deleterious alleles $\left(h s_{d} / s_{h}>1 / 2\right)$ favour haploidy. The strength of this selection on the life cycle (caused by masking alleles) depends on the equilibrium frequency of deleterious alleles, which is greater when the diploid phase is longer (assuming $0<h s_{d} / s_{h}<1$ ).

Using this approximation, haploid-diploid life cycles are evolutionarily singular strategies when $\sigma_{h}-\sigma_{d}=2\left(s_{h}\right) \hat{q}_{a}\left(h_{e}-1 / 2\right)$. Without intrinsic fitness differences, there is no intermediate value of $t_{M M}$ that solves this condition, hence either haplont or diplont life cycles are favoured. Thus, whereas Hall (2000) shows that biphasic haploid-diploid life cycles can evolve if selection occurs once per generation (figure 1a) and mutations occur at meiosis (as considered here), haploid-diploid life cycles in the continuous selection model (figure 1b) do not evolve in the absence of intrinsic fitness differences.

When diploids have higher intrinsic fitness $\left(\sigma_{d}>\sigma_{h}\right)$, there are intermediate (biphasic haploid-diploid) singular strategies in the region where deleterious alleles favour haploidy. In this case, the strength of selection in favour of haploidy is strong when the diploid phase is longer (because deleterious mutations reach higher frequencies) and can outweigh the intrinsic fitness differences. When the diploid phase is short, intrinsic fitness differences dominate, favouring a longer diploid phase. This combination ensures that evolution converges towards a haploid-diploid life cycle (figure 2a).

When haploids have higher intrinsic fitness $\left(\sigma_{h}>\sigma_{d}\right)$, either haplonty or diplonty is always favoured. Even if an intermediate singular strategies exists because deleterious alleles favour diploidy, this is a repelling point, such that either haplonty or diplonty evolves. For these parameters, selection in favour of diplonty is stronger when the diploid phase is longer, favouring even 
longer diploid phases (because the benefits of masking deleterious mutations is greater). Conversely, intrinsic fitness differences dominate when the diploid phase is short, favouring longer haploid phases. Thus haplonty and diplonty can both be stable strategies (figure 2c).

After convergence on a haploid-diploid strategy, we can then ask whether this singular strategy is evolutionarily stable. Using the same weak selection approximations as above, evolutionary stability is given by:

$$
\left.\frac{\delta^{2} \lambda_{l}}{\delta t_{M m}^{2}}\right|_{t_{M m}=t^{*}}=\frac{2\left(-s_{h}\right)\left(\sigma_{d}-\sigma_{h}\right)\left(h s_{d} / s_{h}-1\right)(1-r) w_{a}\left[t^{*}\right] w_{A a}\left[t^{*}\right]}{w_{A}\left[t^{*}\right] w_{A A}\left[t^{*}\right]-(1-r) w_{a}\left[t^{*}\right] w_{A a}\left[t^{*}\right]}
$$

where $t^{*}$ indicates the singular strategy for $t$, the length of the haploid phase. When convergence is stable (requiring that $\sigma_{d}>\sigma_{h}$ and $h s_{d} / s_{h}<1$, see below), the singular strategy is evolutionarily unstable (3 is positive). Thus we expect weak disruptive selection after this singular point is reached. Indeed, our multilocus simulations sometimes displayed branching after 100,000 generations, such that there was a proportion $t^{*}$ of haploid alleles $\left(t_{1}=1\right)$, and a proportion $\left(1-t^{*}\right)$ of diploid alleles $\left(t_{2}=0\right)$. Increasing the number of generations always lead to branching when it was not observed by this time.

The weak selection approximation above assumes that the recombination rate is large relative to selection. Without intrinsic fitness differences, Otto and Goldstein (1992) showed that haploidy is favoured over a larger range of parameter spaces when recombination rates are low because associations between haploid-promoting modifiers and the high fitness, purged genetic backgrounds they create are retained for longer. To consider tighter linkage and/or stronger selection we can use the more accurate expression of $\lambda_{l}$

$$
\lambda_{l}=\exp \left[\left(t_{M m}-t_{M M}\right)\left(\sigma_{h}-\sigma_{d}\right)\right]\left(1+\frac{\mu K_{1}}{K_{2} K_{3}}\right),
$$

where 


$$
\begin{aligned}
K_{1}=1 & -(1-r) \exp \left[-\left(t_{M m}-t_{M M}\right) h s_{d}\right] \\
& -r \exp \left[\left(t_{M m}-t_{M M}\right)\left(s_{h}-h s_{d}\right)\right] \\
& +(1-2 r)\left\{\exp \left[\left(1-t_{M m}-\left(t_{M m}-t_{M M}\right)\right) h s_{d}+t_{M m} s_{h}\right]\right. \\
& \left.\quad-\exp \left[\left(1-t_{M m}\right) h s_{d}+t_{M m} s_{h}\right]\right\} \\
K_{2}=1 & -\exp \left[-\left(1-t_{M M}\right) h s_{d}-t_{M M} s_{h}\right] \\
K_{3}=1- & (1-r) \exp \left[\left(1-t_{M m}\right) h s_{d}+t_{M m} s_{h}\right],
\end{aligned}
$$

in which the per locus mutation rate $(\mu)$ is assumed to be small, so that

terms on the order of the square of the mutation rate can be ignored.

Equation (4) shows that singular strategies can exist without intrinsic fitness differences when recombination rates are low, $r<1 / 2$, see figures $2 \mathrm{~b}$ and $2 \mathrm{~d}$ ). As above, these singular strategies are always repelling points when $\sigma_{d}=\sigma_{h}$ (see supplementary Mathematica file) such that differences in intrinsic fitness are required for haploid-diploid life cycles to evolve. Convergence upon a haploid-diploid life cycle still requires that diploids have higher intrinsic fitness $\left(\sigma_{d}>\sigma_{h}\right.$, see supplementary Mathematica file). However, as selection becomes less weak relative to recombination rates (such that the approximation in 2 is not appropriate), haploid-diploid life cycles can evolve when $h s_{d} / s_{h}<1 / 2$, see figure $2 \mathrm{~b}$. In addition, convergence stability requires $h s_{d} / s_{h}<1$, such that the frequency of deleterious mutations $\left(\hat{q}_{a}\right)$ increases with the length of the diploid phase, see figure $3 \mathrm{a}$.

We next extend our two-locus result to consider deleterious mutations across $L$ viability loci by assuming that these loci are loosely linked, autosomal and nonepistatic. With these assumptions (e.g., Jenkins and Kirkpatrick 1995, Otto and Bourguet 1999, Hough et al. 2013, Rescan et al. 2016), inva- 
sion of a modifier of weak effect is given by

$$
\lambda_{n e t}=1+\sum_{l=1}^{L}\left(\lambda_{l}-1\right) .
$$

332

In figures 2 and $3 a$ we plot where this approximation predicts haplont, diplont or haploid-diploid life cycles to evolve for comparison to the explicit multilocus simulation (described above).

Above, as in previous work, we consider the average dominance and selection coefficients $\left(h, s_{d}\right.$ and $\left.s_{h}\right)$. We can approximate the effect of small amounts of variation (and covariation) among loci in these coefficients by performing a Taylor expansion, as described in Lynch and Walsh (1998), Appendix 1 (see Mathematica file for details). Because we have assumed that deleterious mutations are rare, $s_{d}$ is always found with $h$ and we consider variation in $s_{h}$ and the compound parameter $h s_{d}$. Assuming that deviations between coefficients and their mean value are of order $\epsilon$ and that selection is weak (as assumed in equation 2), yields

$$
\begin{aligned}
\lambda_{n e t} \approx & +\left(t_{M m}-t_{M M}\right)\left(\sigma_{h}-\sigma_{d}+2\left(-s_{h}\right) L \hat{q}_{a}\left(\frac{h s_{d}}{s_{h}}-\frac{1}{2}\right)\right. \\
& +\frac{\left(1+t_{M M}\right) L \hat{q}_{a}\left(-s_{h}\right)}{\mu^{2}}\left(\left(1-t_{M M}\right)\left(\frac{h s_{d}}{s_{h}} \operatorname{Cov}\left(h s_{d}, s_{h}\right)-\operatorname{Var}\left(h s_{d}\right)\right)\right. \\
& \left.+t_{M M}\left(\frac{h s_{d}}{s_{h}} \operatorname{Var}\left(s_{h}\right)-\operatorname{Cov}\left(h s_{d}, s_{h}\right)\right)\right)+O\left(\epsilon^{3}\right)
\end{aligned}
$$

Based on this analysis, variation in $s_{h}$ generally makes haplonty more stable to invasion (reduces $\lambda_{n e t}$ for $t_{M M}=1, t_{M m}<1$ ). Similarly, variation in $h s_{d}$ makes diplonty more stable to invasion (where $t_{M M}=0, t_{M m}>0$ ). Positive covariation between $h s_{d}$ and $s_{h}$ has the opposite effect. Yeast deletion data indicate that the heterozygous effects of deleterious mutations may be much 
less variable than their homozygous effects, due to a negative correlation

between h and s (Phadnis 2005, Agrawal and Whitlock 2011, Manna et al. 2011). Even if $s_{d}$ and $s_{h}$ are on average the same, it may thus be that the variance of $h s_{d}$ is much lower than the variance of $s_{h}$.

\section{Beneficial Mutations}

Whereas deleterious alleles are maintained at mutation-selection balance, beneficial mutations sweep to fixation. The time taken for a sweep to occur depends on the length of the diploid phase; selective sweeps take longer in predominantly diploid populations. During a selective sweep, heterozygotes are present in the population. Life cycle modifiers can affect whether heterozygous zygotes subsequently experience selection as heterozygous diploids or as haploids. Thus, the strength of selection exerted by beneficial mutations on modifiers depends on the time taken for fixation to occur, which depends on the life cycle of the current population. Therefore, as with deleterious alleles, the direction of selection exerted by beneficial mutations depends on dominance. Here we evaluate how these genetic considerations are expected to influence life cycle evolution and include differences in intrinsic fitness between haploids and diploids.

We obtain analytical results using a quasi-linkage equilibrium (QLE) approximation, in which selection is assumed to be weak relative to recombination so that linkage disequilibrium $\left(D=x_{1} x_{4}-x_{2} x_{3}\right)$ equilibrates quickly relative to the rate of change of allele frequencies $\left(p_{A}=x_{1}+x_{3}\right.$ and $\left.p_{M}=x_{1}+x_{2}\right)$. Assuming weak selection, $O(\epsilon)$, and low mutation rates, $O\left(\epsilon^{2}\right)$, the leading order term for the quasi-equilibrium value of linkage disequilibrium $\left(\hat{D}_{Q}\right)$ is given by

$\hat{D}_{Q} \approx \delta_{t} \frac{s_{h}}{r} p_{M}\left(1-p_{M}\right) p_{A}\left(1-p_{A}\right)\left(1-p_{A} \frac{h s_{d}}{s_{h}}-\left(1-p_{A}\right)(1-h) \frac{s_{d}}{s_{h}}\right)+O\left(\epsilon^{2}\right)$ 
where $\delta_{t}=\left(p_{M}\left(t_{M m}-t_{M M}\right)+\left(1-p_{M}\right)\left(t_{m m}-t_{M m}\right)\right)$ is the effect of the modifier on the length of the haploid phase ( $\delta_{t}$ is positive if $m$ increases the haploid phase with $t_{m m}>t_{M m}>t_{M M}$ and negative if $t_{m m}<t_{M m}<t_{M M}$ ).

Linkage disequilibrium is a measure of associations between alleles at different loci. When $D>0$, alleles $A$ and $M$ are more often found together, as are alleles $a$ and $m$. When $s_{h}=s_{d}$ and $0<h<1$, as assumed in Otto (1994) and Orr and Otto (1994), equation (7) shows that $m$ alleles that increase the length of the haploid phase $\left(\delta_{t}>0\right)$ are associated with the beneficial mutation, $a\left(\hat{D}_{Q}>0\right)$. These associations are broken apart by recombination so associations are stronger $\left(\left|\hat{D}_{Q}\right|\right.$ larger $)$ when the recombination rate is low. Therefore lower recombination rates should favour haplonty, as found numerically by Otto (1994) and Orr and Otto (1994).

The change in the frequency of the modifier allele, $m\left(\Delta q_{m}\right)$ can then be expressed as a function of linkage disequilibrium $\left(\hat{D}_{Q}\right)$ and allele frequencies, $p_{A}$ and $p_{M}$. Assuming that selection is weak and mutation rates are low, the leading order term of $\Delta q_{m}$ is given by

$\Delta q_{m} \approx \delta_{t} p_{M}\left(1-p_{M}\right)\left(\sigma_{h}-\sigma_{d}+s_{h}\left(1-p_{A}\right)\left(1-2 p_{A} \frac{h s_{d}}{s_{h}}-\left(1-p_{A}\right) \frac{s_{d}}{s_{h}}\right)\right)+O\left(\epsilon^{2}\right)$.

Unlike deleterious mutations, beneficial mutations reach high frequencies in the population, so the dynamics of the modifier depend on the fitness of both heterozygous and homozygous mutants. Equation (8) shows that, when fixed $\left(p_{A}=0\right)$, a beneficial mutation with a different effect size in haploids and diploids $\left(s_{d} \neq s_{h}\right)$ affects life cycle evolution in a similar manner to intrinsic fitness differences $\left(\sigma_{d}\right.$ and $\left.\sigma_{h}\right)$. However, there is also transient selection on the life cycle that occurs during the fixation of a beneficial mutation. We isolate the transient selection on the life cycle from the effect on intrinsic fitnesses by considering the case where $s_{d}=s_{h}=s$ so that 


$$
\Delta q_{m} \approx \delta_{t} p_{M}\left(1-p_{M}\right)\left(\sigma_{h}-\sigma_{d}+2 p_{A}\left(1-p_{A}\right)(1 / 2-h) s\right)+O\left(\epsilon^{2}\right)
$$

Equation (9) demonstrates that, in the absence of intrinsic fitness differences ${ }_{400}\left(\sigma_{d}=\sigma_{h}\right)$, haplonty is favoured during sweeps of partially recessive $(h<$ $1 / 2$ ) beneficial mutations and diplonty is favoured during sweeps of partially dominant $(h>1 / 2)$ beneficial mutations (as found numerically by Orr and Otto 1994).

404 Whether life cycle evolution is dominated by differences in intrinsic fitness or transient selection generated by beneficial mutations depends on the 406 rate at which beneficial mutations occur and how long they segregate in the population. The fixation time of beneficial mutations is different for different life cycles (longer when diploid phases are longer). We assume that the mutant life cycle allele is rare or similar enough to that of the resident that the time taken to fix a beneficial mutation depends on the life cycle of the resident and then measure the transient selection on the modifier over the 412 entire time course of the sweep using

$$
\int p_{M}\left(1-p_{M}\right) 2 p_{A}\left(1-p_{A}\right) p_{A}(1 / 2-h) s \mathrm{~d} t
$$

This integral can then be evaluated assuming that a beneficial mutation will 414 initially be found at frequency $1 / N$, where $N$ is the population size.

Assuming that the rate of adaptation is limited by the rate of environ416 mental change so that a beneficial mutation fixes every $g$ generations and considering selection on the life cycle from all $L$ loci, the average invasion 418 fitness of a rare life cycle modifier per generation is 


$$
\begin{aligned}
\Delta \bar{q}_{m} \approx & \delta_{t} p_{M}\left(1-p_{M}\right)\left(\left(S_{h}-S_{d}\right)\right. \\
& \left.-\frac{1}{g} \ln \left[\frac{1}{N}+\frac{(N-1)\left(h\left(1-t_{M M}\right)+t_{M M}\right)}{N\left(1-h\left(1-t_{M M}\right)\right)}\right] /\left(1-t_{M M}\right)\right),
\end{aligned}
$$

where the last term accounts for the fact that the beneficial mutations occur only once every $g$ generations.

As with deleterious mutations, there can be haploid-diploid life cycles $\left(0<t_{M M}<1\right)$ that are evolutionarily singular strategies. Assuming that the population size is large, mutants that increase the length of the haploid phase $\left(\delta_{t}>0\right)$ can only invade a resident population that has a short haploid phase $\left(t_{M M}=0\right)$ if beneficial mutations are partially recessive $(0<h<1 / 2)$. Similarly, mutants that decrease the length of the haploid phase $\left(\delta_{t}<0\right)$ can only invade a resident population that has a long haploid phase $\left(t_{M M} \approx 1\right)$ if beneficial mutations are partially recessive $(0<h<1 / 2)$. Therefore, a haploid-diploid life cycle can only be convergence stable when $0<h<1 / 2$ (green in figure $3 \mathrm{~b}$ ). Figure $3 \mathrm{~b}$ also shows the region in which both haplonty and diplonty cannot be invaded by small life cycle modifiers, in which case the singular strategy represents a repelling point (red).

When the rate of adaptation is not limited by the rate of environmental change, but by the rate of fixation of beneficial mutations, the time between fixation events depends on the occurrence of beneficial mutations $(1 / g)$ and their fixation probability $\left(P_{f i x}\right)$, which is given by $2 s\left(t_{M M}+\left(1-t_{M M}\right) h\right)$. Fixation probability decreases when the diploid phase is longer because beneficial mutations are partially hidden by the extra chromosomal copy in diploids. Under mutation-limited adaptation $g$ can be replaced in equation (11) by $g / P_{f i x}$. In this case, haploid-diploid life cycles are never maintained by selection. Thus, beneficial mutations can only favour haploid-diploid life cycles if the rate of adaptation is not mutation-limited. 


\section{Discussion}

444 Empirical evidence suggests that the fitness effects of new mutations are not generally the same in haploids and diploids (Gerstein 2012, Zörgö et al.

446 2013). We show that, when the average fitness effect of new deleterious mutations is unequal in haploids and diploids, whether deleterious mutations favour haploidy or diploidy depends on their effective dominance $\left(h s_{d} / s_{h}\right)$. Most mutation accumulation studies in Saccharomyces yeast estimate either the average heterozygous $\left(h s_{d}\right)$ or haploid $\left(s_{h}\right)$ effect of mutations on fitness (Wloch et al. 2001, Zeyl and DeVisser 2001, Joseph 2004, Hall et al. 2008), from which effective dominance could be estimated. However, because the expectation of a ratio is not generally equal to the ratio of expectations, estimates of effective dominance would be more accurate if calculated from the same strains. In such a study, Korona (1999) took relevant haploid and diploid fitness measures but does not estimate effective dominance. In addition, Szafraniec et al. (2003) found deleterious mutations affected haploid fitness more strongly than diploid fitness but they caution that the haploid spores were required to germinate, which may have biased their fitness measurements in favour of diploids. Thus, further empirical estimates of the effective dominance of deleterious mutations would better inform our understanding of how life cycles are impacted by deleterious mutations.

Haploid and diploid phases can also differ in their intrinsic fitnesses (Thornber 2006, Zörgö et al. 2013). Without differences in intrinsic fitness between haploids and diploids, life cycle evolution depends on the effective dominance of mutations. On the other hand, large differences in intrinsic fitnesses favour expansion of the phase with higher fitness (Jenkins and Kirkpatrick 1994). In this study, we primarily show how life cycles are expected to evolve when both of these selective forces act. To leading order, these selective forces both apply when intrinsic fitness differences are similar in magnitude to the haploid genome-wide mutation rate. For example, figure 3A shows how life cycles are expected to evolve when the deleterious muta- 
tion rate per haploid genome $(U)$ is 0.1 , approximately equal to estimates of

474

476

the deleterious mutation rate in Amsinckia and Arabidopsis plants (Schoen 2005, Halligan and Keightley 2009). Figure 3A suggests that these forces are of similar strength when the intrinsic fitness difference between haploids and diploids $\left(S_{d}-S_{h}\right)$ is between $2 \%$ and $5 \%$. Estimates of the deleterious mutation rate per haploid genome vary across studies and organisms (Halligan and Keightley 2009). For deleterious mutation rates that are a factor $f$ larger, the scale of the $\mathrm{x}$-axis on this figure can be multiplied by $f$ to determine when selection on the life cycle due to deleterious mutations should be approximately the same strength as selection due to differences in intrinsic fitness. We note that mutation rate estimates in yeast and Chlamydomonas (Morgan et al. 2014) are lower but are typically calculated per mitotic cell division. However, the relevant mutation rate for models of life cycle evolution is per sexual cycle (i.e., per meiosis), which has been estimated to involve approximately 1,000 mitotic generations in natural yeast populations (Tsai et al. 2008).

In laboratory environments, substantial differences in fitness between haploid and diploids phases of Saccharomyces yeast and algae have been observed in some environments (Mable and Otto 1998, Destombe et al. 1993, Pacheco-Ruíz et al. 2011, Zörgö et al. 2013). However, measuring the fitness of yeast in natural environments is challenging. Some demographic studies of natural red algae populations of Mazzaella flaccida and Chondrus crispus have shown that diploids have moderately increased survivorship relative to haploids $\left(S_{d}-S_{h} \approx 0.1\right.$, Bhattacharya 1985, Thornber and Gaines 2004). Other studies have found no difference in survivorship, perhaps because there is limited power to detect smaller differences in mortality rates (e.g., Engel et al. 2001, Thornber and Gaines 2004). We also note that, while differences in survivorship of propagules from haploid and diploid phases have been observed (Thornber 2006), this fitness measure is less appropriate because most models assume that both spores and gametes will be produced over the course 
of the life cycle, regardless of the length of the haploid and diploid phases.

Overall, estimates of the magnitude of intrinsic fitness differences are still uncertain, partly because existing algal studies do not compare survivorship of isogenic haploids and diploids, which would be required to remove the effect of masked deleterious mutations in heterozygotes.

For haploid-diploid life cycles to evolve by selection, individuals with longer diploid phases must be favoured in predominantly haploid populations and individuals with longer haploid phases must be favoured in predominantly diploid populations. Previous models predicting the evolution of biphasic haploid-diploid life cycles have posited indirect benefits from decreasing senescence by reducing phase-specific generation time (Jenkins 1993), reducing the frequency of sexual reproduction (Richerd et al. 1993), or exploiting more ecological niches (Bell 1997, Hughes and Otto 1999, Rescan et al. 2016). However, haploid-diploid life cycles are not a unique way of accessing these benefits. For example, diplont or haplont species can reduce generation times or the frequency of sexual reproduction without evolving haploid-diploid life cycles. Similarly, differentiated life cycle stages (Steenstrup alternations), phenotypic plasticity or genetic polymorphism can allow diplontic or haplontic species to exploit multiple ecological niches without tying growth form to the sexual cycle. Here, we use a population genetic model to show that haploid-diploid life cycles can evolve as a direct consequence of ploidy if the intrinsic fitness of haploids and diploids is not equal.

Given that intrinsic fitness differences and genome-wide mutation rates are of a similar magnitude to one another, haploid-diploid life cycles can only evolve in the model presented here if diploids have higher intrinsic fitness than haploids and deleterious/beneficial mutations favour haploidy. In this case, the frequency of deleterious mutations (or time taken for beneficial mutations to fix), and thus the strength selection in favour of haploidy, is largest in predominantly diploid populations and weakest in predominantly haploid populations. In theory, a diploid intrinsic fitness advantage may be 
particularly likely due to several previously proposed hypotheses. Firstly,

534

536

Orr (1995) showed that diplonty can protect organisms from partially recessive somatic mutations (e.g., masking potentially cancerous mutations that arise during development). Although Orr (1995) did not explicitly explore whether haploid-diploid life cycles could evolve, considering somatic mutations that are partially recessive in his model generates a diploid advantage of the type considered here (see Mathematica file). Secondly, Haig and Wilczek (2006) proposed that, when diploid growth is partly provisioned by the female haploid (e.g., if diploids grow on haploids), paternally expressed genes will favour greater female allocation to his diploid offspring, improving the fitness of that phase.

Given that deleterious mutations are typically partially recessive (Simmons and Crow 1977, Agrawal and Whitlock 2011, Manna et al. 2011), the region in which a haploid-diploid life cycle evolves is unlikely to be commonly encountered, except in two circumstances. First, if mutations are more deleterious in homozygous diploids than in haploids $\left(s_{d}>s_{h}\right)$, haploid-diploid life cycles can be favoured when deleterious mutations are partially recessive (figure 2a). Second, when recombination rates are low, the region in which haploid-diploid life cycles are favoured moves into the zone where deleterious mutations are partially recessive (figure $2 b$ ).

A previous investigation by Otto and Marks (1996) found that haploidy was also favoured by recessive deleterious mutations when selfing, asexual reproduction or assortative mating is common (similar to low recombination). These results were interpreted via the fact that these mating schemes partly cause the effective recombination rate to be reduced, e.g., recombination has no impact in a selfed, homozygous individual. However, this analysis assumed that homozygotes and haploids have equal fitness, thus increased homozygosity had no direct impact on fitness. Here, we show that, when haploids and diploids have unequal fitness and/or when new mutations occur during the life cycle (e.g., at meiosis), the net effect of selfing can favour 
haploidy or diploidy (Appendix). We also note that the frequency of delete-

564

rious mutations, and thus their relative impact on life cycle evolution, is also decreased with increased selfing because they are exposed to selection in the homozygous state (Appendix). Thus, if the fitness of haploids and homozygous diploids differs, we caution against generally predicting that haplont and haploid-diploid life cycles should be more common in species where selfing, asexual reproduction and assortative mating are frequent. For example, this may explain why a survey by Mable and Otto (1998) found no correlation between haploidy and the estimated degree of sexuality in protists or green algae.

When the balance between intrinsic fitness differences and the effect of mutations favours convergence on haploid-diploid strategies, disruptive selection then arises such that polymorphisms can evolve with alternative alleles coding for longer haploid and longer diploid phases (i.e., a polymorphic strategy of specialists). In our simulations, a single modifier locus is able to confer fully haplont or diplont life cycles, polymorphism at this locus therefore means that these specialists life cycles can be relatively common (along with the life cycle of the heterozygote at the modifier locus). If genetic control of the life-cycle instead involves many modifier loci, each of which was limited to a having a small effect on the length of the haploid phase, a higher proportion of intermediate phenotypes would be observed in a population experiencing disruptive selection due to mating and recombination. This is especially true when modifier loci are loosely linked because associations between alleles at different loci (linkage disequilibria) are small when recombination is large relative to selection (e.g, Otto and Day 2007, equation 9.45). Disruptive selection was also observed in a density-dependent model where haploids and diploids occupy different niches with or without deleterious mutations (Rescan et al. 2016). Temporal variability of niche sizes can, however, stabilize obligatory alternation between phases (Rescan et al. 2016). Thus, for haploid-diploid life cycles to be favoured over a polymorphic pop- 
ulation of specialist haploids and diploids appears to require constraints on

594

596

the genetic architecture underlying life cycle variation or external variability.

It is intuitively and empirically reasonable that haploids and diploids should both differ in intrinsic fitness and in the extent to which new mutations are masked/revealed to selection. Here, we find the conditions under which these selective forces are approximately balanced and show that this suggests a new hypothesis for the evolution of haploid-diploid life cycles. A significant strength of this hypothesis is that haploid-diploid life cycles evolve in species undergoing an alternation of haploids and diploid phases without positing any extrinsic benefits.

\section{Acknowledgements}

604 We sincerely thank Sally Otto (SPO) for many helpful discussions, guidance and comments on the manuscript and thank Denis Roze for help with multilocus extrapolation analysis, multilocus simulations and helpful comments. Funding for this project was provided by the University of British Columbia and by a Natural Sciences and Engineering Research Council of Canada grant to SPO. 


\section{${ }_{610}$ References}

Agrawal, A. F. and M. C. Whitlock, 2011. Inferences about the distribution

Bell, G., 1994. The comparative biology of the alternation of generations.

— 1997. The evolution of the life cycle of brown seaweeds. Biological

Bhattacharya, D., 1985. The demography of fronds of Chondrus crispus

Stackhouse. Journal of experimental marine biology and ecology 91:217231.

Coelho, S. M., A. F. Peters, B. Charrier, D. Roze, C. Destombe, M. Valero, and J. M. Cock, 2007. Complex life cycles of multicellular eukaryotes: New approaches based on the use of model organisms. Gene 406:152-170.

Crow, J. F. and M. Kimura, 1965. Evolution in sexual and asexual populations. American Naturalist 99:439-450.

Destombe, C., J. Godin, M. Nocher, S. Richerd, and M. Valero, 1993. Differences in response between haploid and diploid isomorphic phases of Gracilaria verrucosa (Rhodophyta: Gigartinales) exposed to artificial environmental conditions. Hydrobiologia 260:131-137.

Engel, C., P. Åberg, O. E. Gaggiotti, and C. Destombe, 2001. Population dynamics and stage structure in a haploid-diploid red seaweed, Gracilaria gracilis. Journal of Ecology 89:436-450.

Gerstein, A. C., 2012. Mutational effects depend on ploidy level: all else is not equal. Biology letters 9:20120614. 
Jenkins, C. D. and M. Kirkpatrick, 1994. Deleterious mutation and ecological 652

Joseph, S. B., 2004. Spontaneous Mutations in Diploid Saccharomyces cere656

Klinger, T., 1993. The persistence of haplodiploidy in algae. Trends in 658 and diploid generations. Philosophical Transactions of the Royal Society

Hall, D. W., 2000. The evolution of haploid, diploid and polymorphic haploiddiploid life cycles: the role of meiotic mutation. Genetics 156:893-898.

Hall, D. W., R. Mahmoudizad, A. W. Hurd, and S. Joseph, 2008. Spontaneous mutations in diploid Saccharomyces cerevisiae: another thousand cell generations. Genetics Research 90:229-241.

Halligan, D. L. and P. D. Keightley, 2009. Spontaneous mutation accumulation studies in evolutionary genetics. Annual Review of Ecology, Evolution, and Systematics 40:151-172.

Hough, J., S. Immler, S. Barrett, and S. P. Otto, 2013. Evolutionarily stable sex ratios and mutation load. Evolution 7:1915-1925.

Hughes, J. and S. Otto, 1999. Ecology and the evolution of biphasic life cycles. The American Naturalist 154:306-320.

Jenkins, C. D., 1993. Selection and the evolution of genetic life cycles. Genetics 133:401-410.

selection in the evolution of life cycles. Lect Math Life Sci 25:53-68.

- 1995. Deleterious mutation and the evolution of genetic life cycles. Evolution 49:512.

visiae: More Beneficial Than Expected. Genetics 168:1817-1825.

Ecology \& Evolution 8:256-258.

Haig, D. and A. Wilczek, 2006. Sexual conflict and the alternation of haploid 
Kondrashov, A. S. and J. F. Crow, 1991. Haploidy or diploidy: which is

660

Korona, R., 1999. Unpredictable fitness transitions between haploid and 662 diploid strains of the genetically loaded yeast Saccharomyces cerevisiae. Genetics 151:77-85.

Lynch, M. and B. Walsh, 1998. Genetics and analysis of quantitative traits. 1 ed. Sinauer Associates.

Mable, B. K., 2001. Ploidy evolution in the yeast Saccharomyces cerevisiae: a test of the nutrient limitation hypothesis. Journal of Evolutionary Biology 14:157-170.

Mable, B. K. and S. P. Otto, 1998. Evolution of alternation of haploid and diploid phases in life cycles. Bioessays 20:453-462.

Manna, F., G. Martin, and T. Lenormand, 2011. Fitness landscapes: an alternative theory for the dominance of mutation. Genetics 189:923-937.

Morgan, A. D., R. W. Ness, P. D. Keightley, and N. Colegrave, 2014. Spontaneous mutation accumulation in multiple strains of the green alga, Chlamydomonas reinhardtii. Evolution 68:2589-2602.

Orr, H. A., 1995. Somatic mutation favors the evolution of diploidy. Genetics 139:1441-1447.

Orr, H. A. and S. P. Otto, 1994. Does diploidy increase the rate of adaptation? Genetics 136:1475-1480.

Otto, S. P., 1994. The role of deleterious and beneficial mutations in the evolution of ploidy levels. Lect Math Life Sci 25:69-96.

Otto, S. P. and D. Bourguet, 1999. Balanced polymorphisms and the evolution of dominance. The American Naturalist 153:561-574. 
Otto, S. P. and T. Day, 2007. A biologist's guide to mathematical modeling in ecology and evolution. Princeton University Pres, Princeton, NJ.

Otto, S. P. and D. B. Goldstein, 1992. Recombination and the evolution of diploidy. Genetics 131:745-751.

Otto, S. P. and J. C. Marks, 1996. Mating systems and the evolutionary transition between haploidy and diploidy. Biological Journal of the Linnean Society 57:197-218.

Pacheco-Ruíz, I., A. Cabello-Pasini, J. A. Zertuche-González, S. Murray, J. Espinoza-Avalos, and M. J. Dreyfus-Leon, 2011. Carpospore and tetraspore release and survival in Chondracanthus squarrulosus (Rhodophyta: Gigartinaceae) from the Gulf of California. Botanica Marina 54:127-134.

Perrot, V., S. Richerd, and M. Valero, 1991. Transition from haploidy to diploidy. Nature 351:315-317.

Phadnis, N., 2005. Widespread correlations between dominance and homozygous effects of mutations: Implications for theories of dominance. Genetics 171:385-392.

Rescan, M., T. Lenormand, and D. Roze, 2016. Interactions between genetic and ecological effects on the evolution of life cycles, vol. 187. The American Naturalist.

Richerd, S., D. Couvet, and M. Valero, 1993. Evolution of the alternation of haploid and diploid phases in life cycles. II. Maintenance of the haplodiplontic cycle. Journal of Evolutionary Biology 6:263-280.

Schoen, D. J., 2005. Deleterious mutation in related species of the plant genus Amsinckia with contrasting mating systems. Evolution 59:2370-2377. 
Simmons, M. J. and J. F. Crow, 1977. Mutations affecting fitness in Drosophila populations. Annual Review of Genetics 11:49-78.

Szafraniec, K., D. M. Wloch, P. Sliwa, R. H. Borts, and R. Korona, 2003. ${ }_{712}$ Small fitness effects and weak genetic interactions between deleterious mutations in heterozygous loci of the yeast Saccharomyces cerevisiae. Genetic Research 82:19-31.

Thornber, C. S., 2006. Functional properties of the isomorphic biphasic algal 716 life cycle. Integrative and Comparative Biology 46:605-614.

Thornber, C. S. and S. D. Gaines, 2004. Population demographics in species 718 with biphasic life cycles. Ecology 85:1661-1674.

Tsai, I. J., D. Bensasson, A. burt, and V. Koufopanou, 2008. Population 720 genomics of the wild yeast Saccharomyces paradoxus: Quantifying the life cycle. Proc Natl Acad Sci 105:4957-4962.

722

Valero, M., S. Richerd, V. Perrot, and C. Destombe, 1992. Evolution of alternation of haploid and diploid phases in life cycles. Trends in Ecology \& Evolution 7:25-29.

Wloch, D. M., K. Szafraniec, R. H. Borts, and R. Korona, 2001. Direct estimate of the mutation rate and the distribution of fitness effects in the yeast Saccharomyces cerevisiae. Genetics 159:441-452.

Wolfram Research Inc., 2010. Mathematica. Version 8.0 ed. Wolfram Research, Inc., Champaign, Illinois.

Zeyl, C. and J. A. G. M. DeVisser, 2001. Estimates of the Rate and Distribution of Fitness Effects of Spontaneous Mutation in Saccharomyces cerevisiae. Genetics 157:53-61. 
Zörgö, E., K. Chwialkowska, A. B. Gjuvsland, E. Garré, P. Sunnerhagen, ${ }_{734}$ G. Liti, A. Blomberg, S. W. Omholt, and J. Warringer, 2013. Ancient evolutionary trade-offs between yeast ploidy states. PLOS Genetics 736 9:e1003388. 

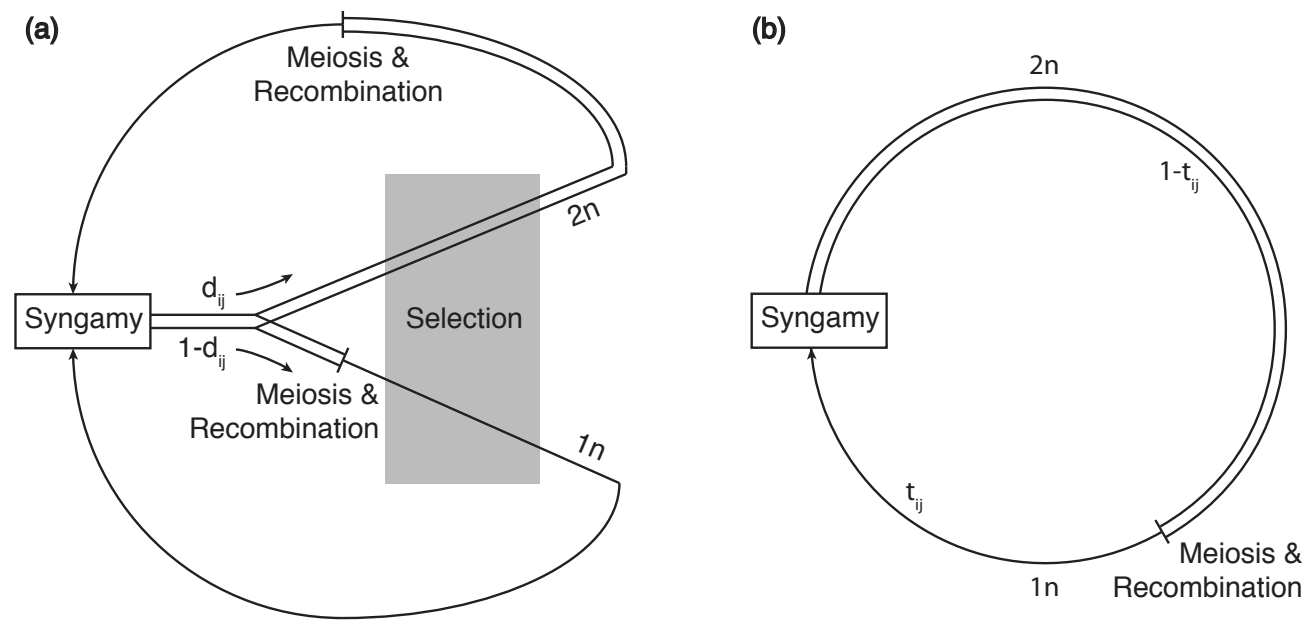

Figure 1: Model (a) discrete selection and (b) continuous selection haploid-diploid life cycles. Single lines represent haploid phases and doubled lines indicate diploid phases. In (a), modified from Perrot et al. (1991) and Otto and Goldstein (1992), zygotes with the modifier genotype $i j$ undergo selection as diploids with probability $d_{i j}$ or undergo meiosis and recombination before experiencing selection as haploids with probability $\left(1-d_{i j}\right)$. In (b), after Jenkins and Kirkpatrick (1994; 1995) and Otto (1994), all zygotes with genotype $i j$ experience viability selection as a diploid for a proportion $\left(1-t_{i j}\right)$ of their life cycle before undergoing meiosis and recombination and then experiencing viability selection as a haploid for the remainder of the life cycle. 

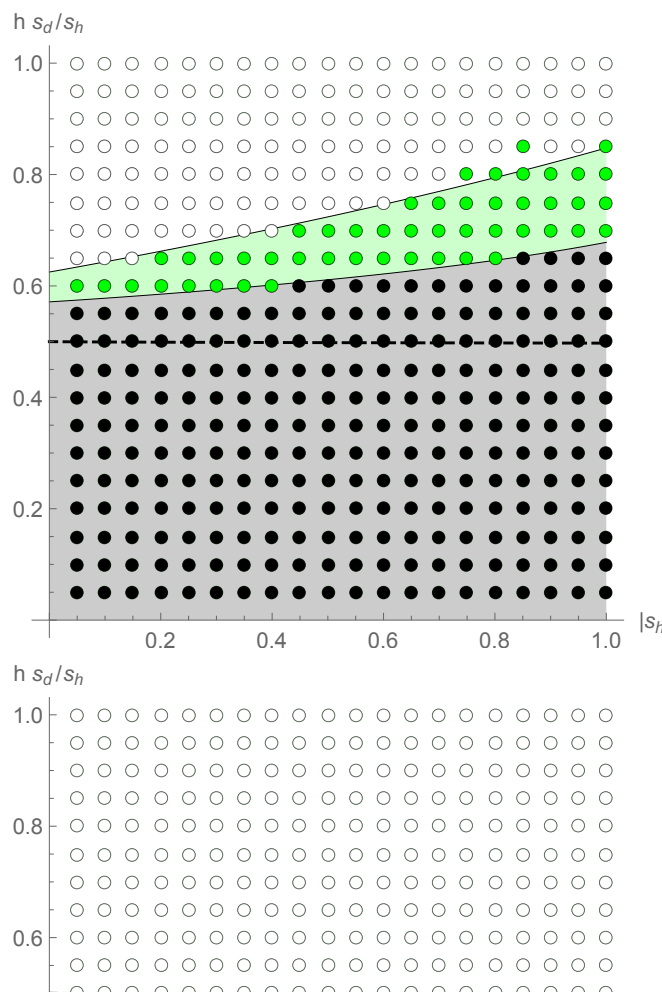

$$
\text { h }
$$

$\mathrm{h} s_{d} / s_{h}$

1.000000000000000000000

0.8

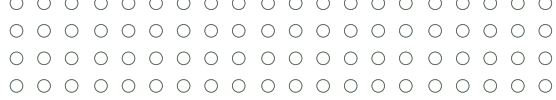

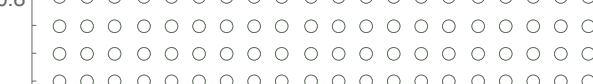

0.6

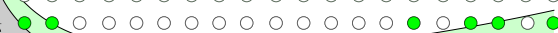

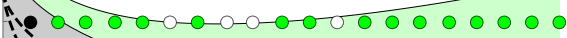

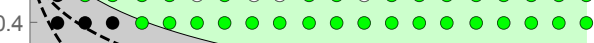

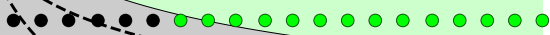

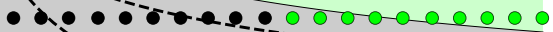
-

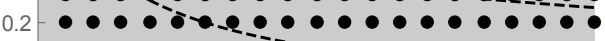

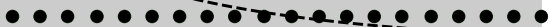

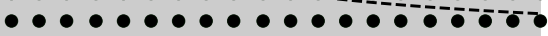

$\bullet \bullet \bullet \bullet \bullet \bullet \bullet \bullet \bullet \bullet \bullet \bullet \bullet \bullet \bullet \bullet \bullet \bullet \bullet$

0.2

0.4

0.6

0.8

$\mathrm{h} s_{d} / s_{h}$

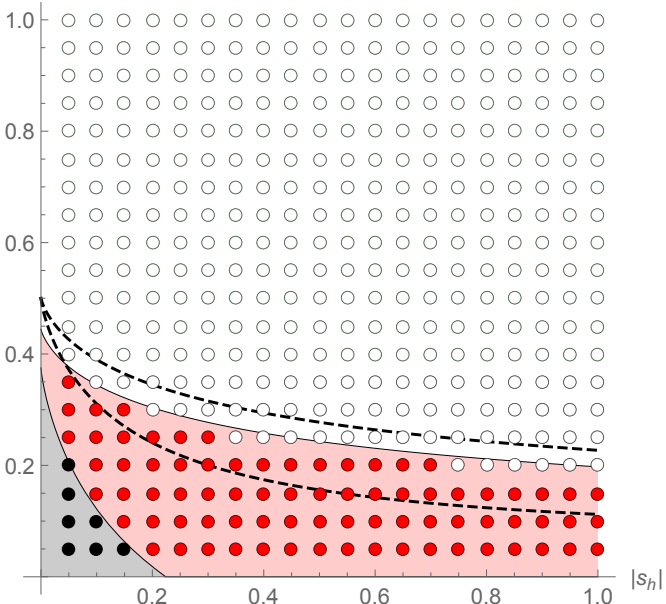

0.2

0.4

0.6

0.8

1.0

Figure 2: Parameter space where haplont, diplont and haploid-diploid life cycles are favoured where the strength of selection against deleterious mutations $\left(\left|s_{h}\right|\right)$ and effective dominance $h s_{d} / s_{h}$ is varied. Background colors: prediction from the two-locus stability analysis extrapolated to multiple loci. Circles: multilocus simulation results starting from three different initial haploidy rates $\left(t_{\text {init }}=0.01,0.5\right.$, or 0.99$)$, with population size 20,000. White: evolution toward haplonty. Green: convergence stable haploid-diploid life cycles. Red: either haplonty or diplonty is favoured, with a repelling state in between. Black and gray: evolution toward diplonty. (a) and (b): diploids have higher intrinsic fitness $\left(S_{h}=0, S_{d}=0.025\right)$ (c) and (d): haploids have higher intrinsic fitness $\left(S_{h}=0.025, S_{d}=0\right)$. Map length: $R=100((\mathrm{a})$ and (c)) and $R=0.35((\mathrm{~b})$ and $(\mathrm{d})$ ). The dashed lines show where haplonty (above dashed lines) and diplonty (below dashed lines) are favoured when there is no difference in intrinsic fitness $\left(S_{h}=S_{d}=0\right)$. In (b) and (d), there is a repelling point between the dashed lines. Mutants change the life cycle by a small amount $\left(\left|t_{M m}-t_{M M}\right|=0.001\right)$ and the genome-wide haploid mutation rate, $U=0.1$. 


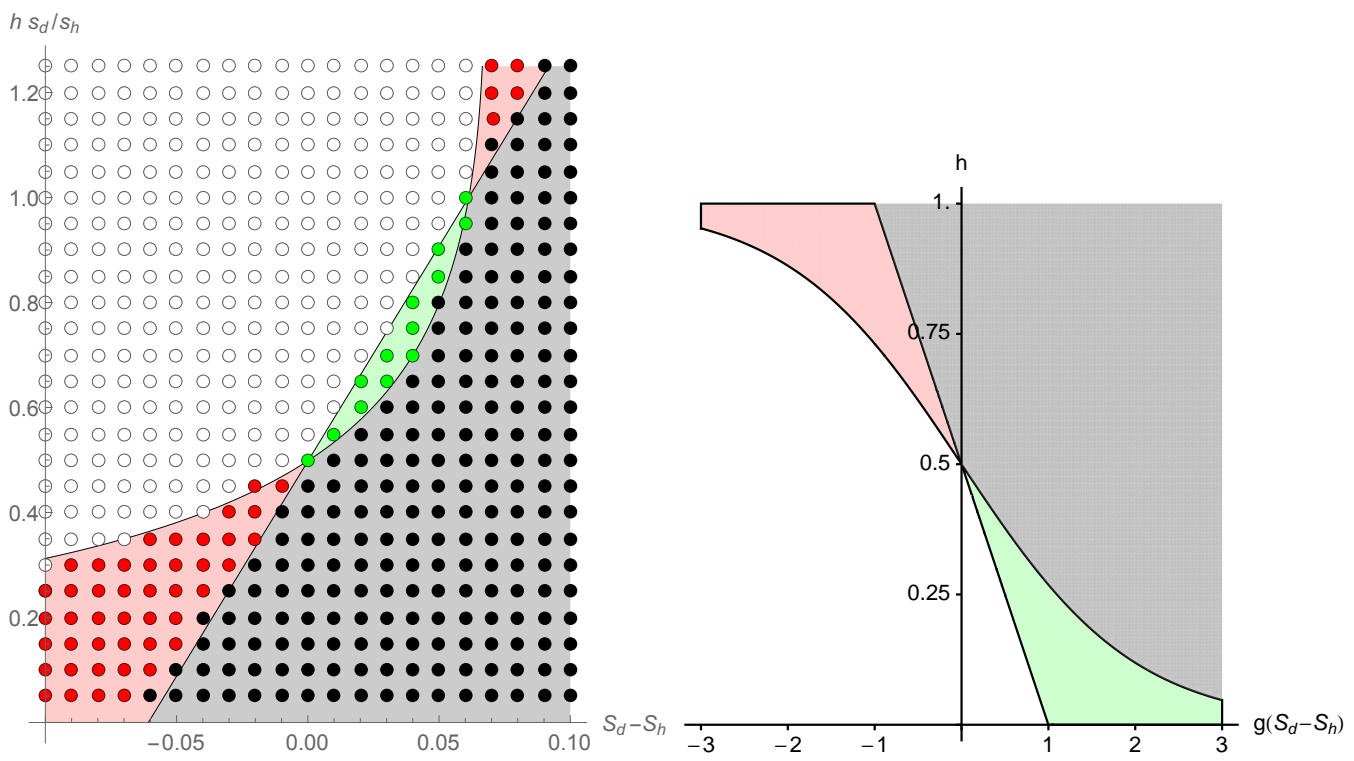

Figure 3: Parameter space for which (a) deleterious mutations and (b) beneficial mutations favour haplont, diplont and haploid-diploid life cycles as a function of the difference in intrinsic fitness between haploids and diploids $\left(S_{d}-S_{h}\right)$. (a) Shows the effective dominance of deleterious mutations $\left(h s_{d} / s_{h}\right)$ against intrinsic fitness differences $\left(S_{d}-S_{h}\right)$, parameters and symbols as in figures 2a and 2c with $\left|s_{h}\right|=0.4$. (b) Regions in which particular life cycles are favoured in the presence of beneficial mutations, evaluated using equation 11. $g$ is the number of generations between fixation events. Population size, $N$, is 20000. 


\section{Appendix}

738

We consider four models: two continuous selection models and two discrete selection models with mutations occurring at either meiosis or gamete production. We allow selfing to occur among gametes at rate $\sigma$, following Otto and Marks (1996). In the main text, we primarily discuss the continuous selection model with mutations at meiosis where $\sigma=0$. We denote the genotypes $M A, M a, m A$ and $m a$ by indices 1 to 4 , the frequency of these genotypes in the next generation $x_{1}^{\prime}, x_{2}^{\prime}, x_{3}^{\prime}$ and $x_{4}^{\prime}$ ) are given by

$$
\begin{gathered}
x_{1}^{\prime}=(1-\mu)\left((1-\sigma)\left(x_{1}^{2} w_{11, A}+x_{1} x_{2} w_{12, A}+x_{1} x_{3} w_{13, A}+x_{1} x_{4} w_{14, A}-r D w_{14, A}\right)\right. \\
\left.+\sigma x_{1} w_{11, A}\right) / \bar{W}
\end{gathered}
$$

$$
\begin{aligned}
x_{2}^{\prime}=((1-\sigma) & \left(x_{2} x_{1} w_{12, a}+x_{2}^{2} w_{22, a}+x_{2} x_{3} w_{23, a}+x_{2} x_{4} w_{24, a}+r D w_{14, a}\right) \\
& +\sigma x_{2} w_{22, a} \\
+\mu & \left((1-\sigma)\left(x_{1}^{2} w_{11, A \mu}+x_{1} x_{2} w_{12, A \mu}+x_{1} x_{3} w_{13, A \mu}+x_{1} x_{4} w_{14, A \mu}-r D w_{14, A \mu}\right)\right. \\
& \left.\left.+\sigma x_{1} w_{11, A \mu}\right)\right) / \bar{W}
\end{aligned}
$$

$x_{3}^{\prime}=(1-\mu)\left((1-\sigma)\left(x_{3} x_{1} w_{13, A}+x_{3} x_{2} w_{23, A}+x_{3}^{2} w_{33, A}+x_{3} x_{4} w_{34, A}-r D w_{14, A}\right)\right.$

$$
\left.+\sigma x_{3} w_{33, A}\right) / \bar{W}
$$

$$
\begin{aligned}
& x_{4}^{\prime}=\left((1-\sigma)\left(x_{4} x_{1} w_{14, a}+x_{4} x_{2} w_{24, a}+x_{4} x_{3} w_{34, a}+x_{4}^{2} w_{44, a}+r D w_{14, a}\right)\right. \\
& +\sigma x_{4} w_{44, a} \\
& +\mu\left((1-\sigma)\left(x_{3} x_{1} w_{13, A \mu}+x_{3} x_{2} w_{23, A \mu}+x_{3}^{2} w_{33, A \mu}+x_{3} x_{4} w_{34, A \mu}-r D w_{14, A \mu}\right)\right. \\
& \left.\left.\quad+\sigma x_{3} w_{33, A \mu}\right)\right) / \bar{W}
\end{aligned}
$$


where $D=x_{1} x_{4}-x_{2} x_{3}$ and $\bar{W}$ is the sum of the numerators. The notation $w_{i j, k}$ refers to the fitness of a zygote formed by gametes with indices $i$ and $j$ that produces a haploid of type $k$ without mutation, $w_{i j, k \mu}$ is similar but where the $k$ haploid produced by meiosis mutates. These fitnesses for the discrete and continuous selection models are given in table S.1. When mutations occur at gamete production, mutation does not affect fitness and $w_{i j, A \mu}=w_{i j, A}$. The fitness values where mutations occur at meiosis are given in table S.2.

Table S.1: Fitnesses in discrete and continuous selection models.

\begin{tabular}{lll}
\hline \hline Fitness & Continuous selection & Discrete selection \\
\hline$w_{11, A}$ & $w_{A A}\left(t_{M M}\right) w_{A}\left(t_{M M}\right)$ & $w_{A A} d_{M M}+w_{A}\left(1-d_{M M}\right)$ \\
$w_{12, A}$ & $w_{A a}\left(t_{M M}\right) w_{A}\left(t_{M M}\right)$ & $w_{A a} d_{M M}+w_{A}\left(1-d_{M M}\right)$ \\
$w_{12, a}$ & $w_{A a}\left(t_{M M}\right) w_{a}\left(t_{M M}\right)$ & $w_{A a} d_{M M}+w_{a}\left(1-d_{M M}\right)$ \\
$w_{13, A}$ & $w_{A A}\left(t_{M m}\right) w_{A}\left(t_{M m}\right)$ & $w_{A A} d_{M m}+w_{A}\left(1-d_{M m}\right)$ \\
$w_{14, A}=w_{23, A}$ & $w_{A a}\left(t_{M m}\right) w_{A}\left(t_{M m}\right)$ & $w_{A a} d_{M m}+w_{A}\left(1-d_{M m}\right)$ \\
$w_{14, a}=w_{23, a}$ & $w_{A a}\left(t_{M m}\right) w_{a}\left(t_{M m}\right)$ & $w_{A a} d_{M m}+w_{a}\left(1-d_{M m}\right)$ \\
$w_{22, a}$ & $w_{a a}\left(t_{M M}\right) w_{a}\left(t_{M M}\right)$ & $w_{a a} d_{M M}+w_{a}\left(1-d_{M M}\right)$ \\
$w_{24, a}$ & $w_{a a}\left(t_{M m}\right) w_{a}\left(t_{M m}\right)$ & $w_{a a} d_{M m}+w_{a}\left(1-d_{M m}\right)$ \\
$w_{33, A}$ & $w_{A A}\left(t_{m m}\right) w_{A}\left(t_{m m}\right)$ & $w_{A A} d_{m m}+w_{A}\left(1-d_{m m}\right)$ \\
$w_{34, A}$ & $w_{A a}\left(t_{m m}\right) w_{A}\left(t_{m m}\right)$ & $w_{A a} d_{m m}+w_{A}\left(1-d_{m m}\right)$ \\
$w_{34, a}$ & $w_{A a}\left(t_{m m}\right) w_{a}\left(t_{m m}\right)$ & $w_{A a} d_{m m}+w_{a}\left(1-d_{m m}\right)$ \\
$w_{44, a}$ & $w_{a a}\left(t_{m m}\right) w_{a}\left(t_{m m}\right)$ & $w_{a a} d_{m m}+w_{a}\left(1-d_{m m}\right)$ \\
\hline & &
\end{tabular}

Table S.2: Fitnesses of mutated types when mutations occur at meiosis.

\begin{tabular}{lll}
\hline \hline Fitness & Continuous selection & Discrete selection \\
\hline$w_{11, A \mu}$ & $w_{A A}\left(t_{M M}\right) w_{a}\left(t_{M M}\right)$ & $w_{A A} d_{M M}+w_{a}\left(1-d_{M M}\right)$ \\
$w_{12, A \mu}$ & $w_{A a}\left(t_{M M}\right) w_{a}\left(t_{M M}\right)$ & $w_{A a} d_{M M}+w_{a}\left(1-d_{M M}\right)$ \\
$w_{13, A \mu}$ & $w_{A A}\left(t_{M m}\right) w_{a}\left(t_{M m}\right)$ & $w_{A A} d_{M m}+w_{a}\left(1-d_{M m}\right)$ \\
$w_{14, A \mu}=w_{23, A \mu}$ & $w_{A a}\left(t_{M m}\right) w_{a}\left(t_{M m}\right)$ & $w_{A a} d_{M m}+w_{a}\left(1-d_{M m}\right)$ \\
$w_{33, A \mu}$ & $w_{A A}\left(t_{m m}\right) w_{a}\left(t_{m m}\right)$ & $w_{A A} d_{m m}+w_{a}\left(1-d_{m m}\right)$ \\
$w_{34, A \mu}$ & $w_{A a}\left(t_{m m}\right) w_{a}\left(t_{m m}\right)$ & $w_{A a} d_{m m}+w_{a}\left(1-d_{m m}\right)$ \\
\hline
\end{tabular}


We then calculate the frequency of the $a$ allele $\left(\hat{q}_{a}\right)$ when the modifier locus is fixed for a resident allele, $M$, which is given by

$$
\hat{q}_{a}=\frac{\mu w_{11, A \mu}}{w_{11, A}-(1-\sigma) w_{12, a}-\sigma w_{22, a}},
$$

where we ignore terms on the order of $\mu^{2}$. For the continuous selection model with mutations at meiosis and $\sigma=0$, this is equivalent to equation (1). As in the main text, we then evaluate the spread of a rare modifier using the leading eigenvalue $\left(\lambda_{l}\right)$ of the system described by equations A.1c and A.1d. Full expressions of $\lambda_{l}$ for each of the life cycles considered can be found in the supplementary Mathematica notebook.

In the models in which mutations occur at gamete production, and assuming that the fitnesses of $A$ haploids and $A A$ diploids are equal (such that $\left.w_{11, A}=w_{13, A}=w_{33, A}=1\right)$, invasion occurs $\left(\lambda_{l}>1\right)$ if

$$
\begin{aligned}
0< & \sigma\left(w_{22, a}-w_{44, a}\right)\left(w_{12, A}-w_{14, A}(1-r)\right) \\
& +r(1-\sigma)\left(w_{12, A} w_{14, a}+w_{14, A}\left(w_{12, a}-2 w_{14, a}\right)\right. \\
& +\left(w_{12, A}-w_{14, A}\right)\left(1-w_{14, a}(1-\sigma)-w_{22, a} \sigma\right) .
\end{aligned}
$$

Increased selfing can either increase or decrease the parameter range over which this inequality is satisfied unless it is further assumed that the fitness of $a$ haploids and $a a$ diploids are equal (such that $w_{22, a}=w_{44, a}$ and the first term in A.3 is 0 ).

When the fitnesses of haploids and homozygous diploids are equal and mutations occur at gamete production, Otto and Marks (1996) showed that haploidy is always favoured over a larger parameter space when selfing is higher in the discrete selection model. Similarly, in the continuous selection model, where we also assume that modifiers have a small effect, $t_{M m}-t_{M M}=$ $\delta_{t M m}$ is of order $\mu$, modifiers that increase the length of the haploid phase $\left(\delta_{t M m}>0\right)$ invade if 


$$
\begin{array}{r}
h\left(w_{A A}\left(t_{M M}\right) w_{A}\left(t_{M M}\right)-(1-\sigma) w_{A a}\left(t_{M M}\right) w_{a}\left(t_{M M}\right)-\sigma w_{a a}\left(t_{M M}\right) w_{a}\left(t_{M M}\right)\right) \\
>r(1-\sigma)(1-2 h) w_{a}\left(t_{M M}\right) w_{A A}\left(t_{M M}\right) .
\end{array}
$$

776

This condition is always met when $h>1 / 2$ and is always satisfied for a greater parameter range with higher selfing rates (higher $\sigma$ ) if $h<1 / 2$.

In the continuous selection model with mutations at meiosis, however, the impact of selfing is not so simple. Even when we assume the fitnesses of haploids and homozygous diploids is equal $\left(s_{h}=s_{d}\right.$ and $\left.\sigma_{d}=\sigma_{h}=0\right)$ and modifiers have a small effect $\left(t_{m m}-t_{M M}=\delta_{t m m}\right.$ and $t_{M m}-t_{M M}=h_{m} \delta_{t m m}$, where $\delta_{t m m}$ is of order $\mu$ and terms of $O\left(\mu^{2}\right)$ are discarded) and make the further assumption that recombination is free $(r=1 / 2)$, haploidy is favoured when

$$
h>\frac{1-\left(1-h_{m}\right)(1-\sigma)\left(1+\sigma w_{a}\left(t_{M M}\right) w_{A a}\left(t_{M M}\right) / K_{1}\right)}{2 h_{m}},
$$

where $K_{1}=w_{A A}\left(t_{M M}\right) w_{A}\left(t_{M M}\right)-\sigma w_{a a}\left(t_{M M}\right) w_{a}\left(t_{M M}\right)$. For dominant modifiers $\left(h_{m}=1\right)$, this condition is satisfied if and only if $h>1 / 2$, such that selfing has no effect on whether haploidy or diploidy is favoured. When $0<h_{m}<1$, increased selfing increases the right hand side of inequality (A.5). Therefore, increased selfing decreases, rather than increases, the parameter range under which haploidy is favoured. Although selfing can facilitate the evolution of haploidy when $r<1 / 2$ (presumably because the impact of disequilibrium is greater), our overall finding is that when mutations occur at meiosis, selfing does not uniformly favour haploidy even when we assume that the fitness of haploids and homozygous diploids are equal.

In addition, the convergence properties of discrete and continuous selection models differ. For example, Hall (2000) found that, without selfing or 
intrinsic fitness differences, haploid-diploid life cycles can evolve in the discrete selection model where mutations occur at meiosis. However, in the main text we show that haploid-diploid life cycles do not evolve in the continuous selection model where mutations occur at meiosis without intrinsic fitness differences. For the purposes of this study, one important distinction between models is whether haploid-diploid life cycles evolve for recessive deleterious mutations with selfing and loose linkage $(\sigma>0, r=1 / 2)$. In figure S.1, we show a numerical example of life cycle evolution with selfing, loose linkage, and $s_{d}=s_{h}$. For these parameters, haploid-diploid life cycles evolve for low $h$ in the discrete selection model but not in the continuous selection model (where mutations occur at gamete production in both cases). Thus in both the case considered by Hall (2000) (mutations at meiosis with no selfing) and in figure S.1 (mutations at gamete production with selfing), life-cycle models in which selection occurs continously (figure 1b) favour haploid-diploid life cycles less often than discrete life cycle models (figure 1a)

Finally, we clarify how selfing affects the disequilibrium between the $M$ and $A$ loci, which was discussed in Otto and Marks (1996). Using the same model and assumptions as Otto and Marks (1996), where $w_{A A}=w_{A}=1$, $w_{A a}=1-h s$, and $w_{a}=w_{a a}=1-s$ we find that the disequilibrium, $D=x_{1} x_{4}-x_{2} x_{3}$ during invasion of a modifier is given by

$$
D=\frac{\left(d_{M m}-d_{m m}\right)(1-h) \mu(1-\sigma)}{K_{5}\left(1-d_{M M}(1-h)(1-\sigma)\right)}
$$

where $K_{5}=r(1-\sigma)+s\left(1-d_{M m}\right)(1-h)(1-r)+h s(1-r)(1-\sigma)+\sigma s$ is strictly positive. Thus, disequilibrium has the same sign as $\left(d_{M m}-d_{M M}\right)$ and is positive for modifiers that increase the the diploid phase (modifiers associated with the less fit allele) and negative for modifiers that increase the haploid phase, as found by Otto and Marks (1996). However, the magnitude of this disequilibrium decreases with increasing selfing, contrary to the result stated in Otto and Marks (1996). In the supplementary Mathematica file we show that the magnitude of the disequilibrium increases with increasing 
selfing if $\hat{q}_{a}$ is held constant but because selfing also helps purging and reduces
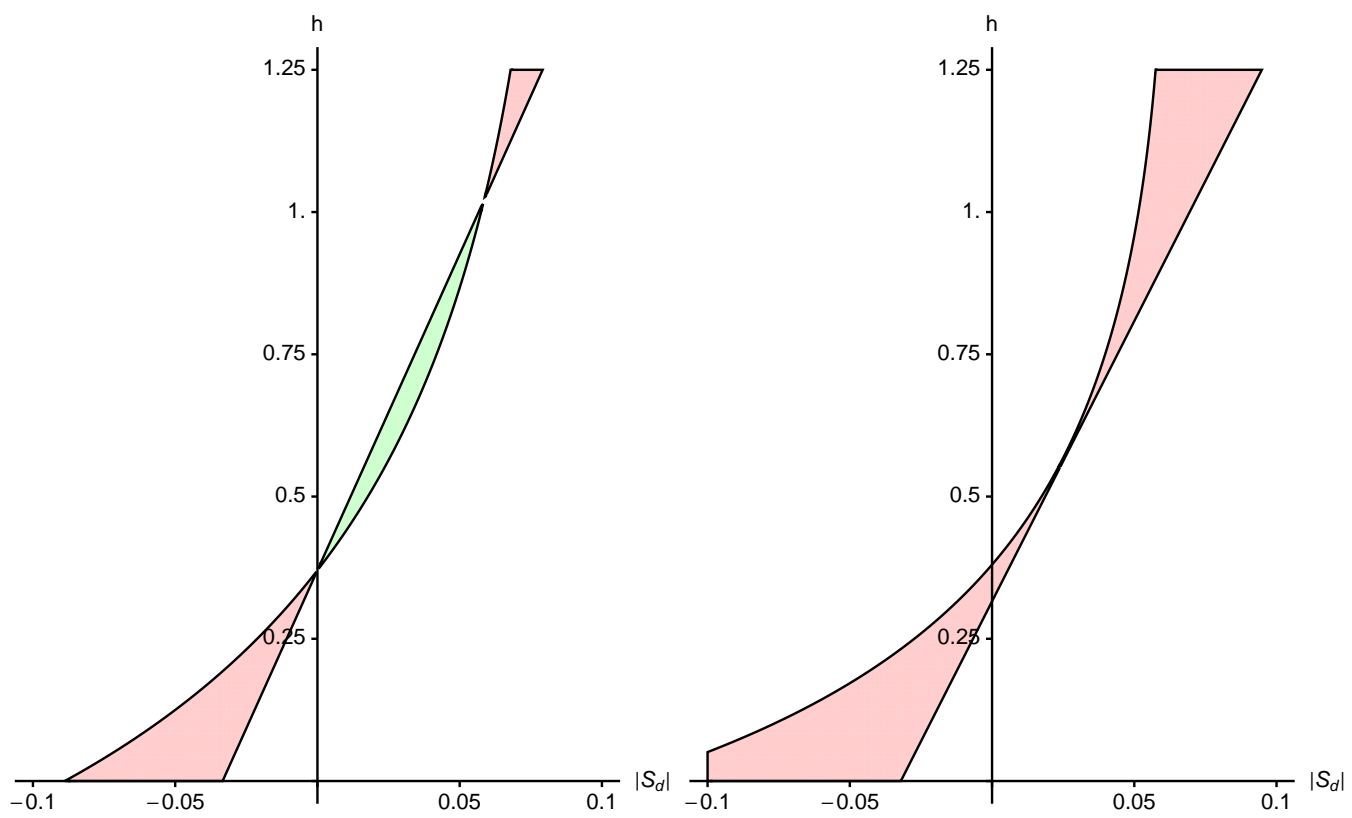

Figure S.1: Here we plot whether haplont, diplont, or haploid-diploid life cycles are favoured when there is selfing among gametes as a function of the intrinsic fitness of diploids $\left(S_{d}\right)$ for (a) the discrete selection model with mutations at gamete production and (b) the continuous selection model with mutations at gamete production. To evaluate expected life cycle evolution we evaluated the stability of pure haplont $\left(d_{M M}=0, t_{M M}=1\right)$ or diplont $\left(d_{M M}=1, t_{M M}=0\right)$ strategies using equation (5) with the full expression of $\lambda_{l}$ where terms on the order of $\mu^{2}$ are discarded, which can be found in the supplementary Mathematica file. In both plots $\sigma=0.4, r=1 / 2, s_{d}=s_{h}=-0.3, U=0.1, L=1000, S_{h}=0$, and modifiers have a small and dominant effect $\left(t_{m m}=t_{M m},\left|t_{M m}-t_{M M}\right|=1 / 10,000, d_{m m}=d_{M m}\right.$, $\left.\left|d_{M m}-d_{M M}\right|=1 / 10,000\right)$. 